\title{
H loss mechanism during anneal of silicon nitride: Chemical dissociation
}

\author{
Christoph Boehme ${ }^{\mathrm{a})}$ and Gerald Lucovsky \\ Department of Physics, North Caroline State University, Raleigh, North Carolina 27695-8202
}

(Received 13 March 2000; accepted for publication 7 September 2000)

\begin{abstract}
Remote plasma enhanced chemical vapor deposited silicon nitride $\left(\mathrm{Si}_{x} \mathrm{~N}_{y} \mathrm{H}_{z}\right)$, produced at high ammonia to silane flow rates (ammonia rich) shows a reduction of hydrogen during rapid thermal anneal at temperatures that exceed the deposition temperature. This $\mathrm{H}$ release could be either due to a "slow" atomic diffusion of the covalent bonded atoms between bonding sites, or to a "fast" molecular diffusion of hydrogen containing molecules (e.g., $\mathrm{H}_{2}, \mathrm{NH}_{3}, \mathrm{SiH}_{4}$ ), which dissociate from the network before they diffuse. In order to determine the dominant mechanism, layers of deuterated and hydrogenated silicon nitride on top of a crystalline $\mathrm{Si}$ substrate were annealed and the development of the NH- and ND-area densities were measured with Fourier transform infrared spectroscopy. Comparison of theoretical models with the measurements showed that chemical dissociation and subsequent rapid diffusion are the dominant processes. These results were confirmed by secondary ion mass spectroscopy. The experiments indicate that the $\mathrm{H}$ reduction in silicon nitride antireflection coatings of solar cells is mostly due to $\mathrm{H}$ migration out of the system and not into the $\mathrm{Si}$ area and make the hypothesis that postdeposition annealing of solar cell antireflection coatings can cause H-related bulk passivation of the underlying $c$-Si therefore questionable. () 2000 American Institute of Physics. [S0021-8979(00)09423-8]
\end{abstract}

\section{INTRODUCTION}

It has been confirmed several times in recent years that the postdeposition anneal of amorphous silicon nitride antireflection coatings increases the internal quantum efficiency of underlying Si solar cells. ${ }^{1-3}$ If this is caused by hydrogen depends mostly on whether the hydrogen is released from the silicon nitride into the $c$-Si bulk or only into the environment of the system. Therefore it is important to know the mechanism responsible for the $\mathrm{H}$ release.

Bik et al. ${ }^{4}$ observed a "slow" $\mathrm{H}$ diffusion in low pressure chemical vapor deposited (LPCVD) silicon nitride with a low diffusion constant with high activation energy of $2.95 \pm 0.15 \mathrm{eV}$. The proposed mechanism is an atomic diffusion of $\mathrm{H}$ atoms that jump between covalent $\mathrm{NH}$ bonds. In addition to the $\mathrm{H}$ transport by atomic diffusion, hydrogen can also migrate through silicon nitride while it is attached to small molecules like molecular hydrogen, ammonia, or silane. These molecules have small activation energies and therefore high diffusivities at high temperatures. However, unlike atomic $\mathrm{H}$ diffusion, this process requires a preceding chemical reaction during which the small molecules dissociate from the network. ${ }^{5}$ Since the dissociation energies and the activation energy of the atomic diffusion process have the same order of magnitude (eV range) the overall H-bond density in a given film is reduced for both mechanisms in a similar way making it difficult to distinguish the two effects and their consequences on the $\mathrm{H}$ reduction during the anneal. Since the possibility of $\mathrm{H}$ permeation into the $c$-Si substrate depends on the $\mathrm{H}$ density at the interface, the type of $\mathrm{H}$ transport mechanism in the silicon nitride is of crucial importance.

\footnotetext{
a)Electronic mail: boehme@hmi.de
}

\section{EXPERIMENT}

An experiment was developed that allowed one to distinguish atomic and molecular transport processes of hydrogen in silicon nitride, where most of the observation was done with Fourier transform infrared (FTIR) spectroscopy while secondary ion mass spectroscopy (SIMS) was only employed as a supporting spectroscopy on a few samples.

For the test of the atomic diffusion, a stack system was deposited (Fig. 1). It contained a $c$-Si substrate, a thin layer of silicon dioxide, a $400 \AA$ A layer of deuterated silicon nitride $\left(\mathrm{Si}_{x} \mathrm{~N}_{y} \mathrm{D}_{z}\right)$, and a $400 \AA$ layer of hydrogenated silicon nitride $\left(\mathrm{Si}_{x} \mathrm{~N}_{y} \mathrm{H}_{z}\right)$. The oxide layer thicknesses were 4,10 , and $20 \AA$.

The stack systems were deposited by remote plasma enhanced CVD (RPECVD) in a flowing afterglow reactor configuration $^{6,7}$ at a temperature of $200^{\circ} \mathrm{C}$ and with an ammonia to silane ratio of 20 , which causes a high $\mathrm{NH}$ and a low $\mathrm{SiH}$ content and therefore a low index of refraction which is wanted for good antireflective (AR) coatings. After the deposition, a $20 \mathrm{~s}$ rapid thermal anneal (RTA) in an $\mathrm{Ar}$ environment was carried out.

\section{FTIR AND SIMS MEASUREMENTS}

FTIR was used to determine the postanneal bond content of ND and NH for the 15 diffusion samples (three oxide thicknesses times five temperatures between 500 and $900^{\circ} \mathrm{C}$ ). Quantification was carried out by integration of the stretching mode absorbance peaks of ND bonds $(\approx 2479$ $\left.\mathrm{cm}^{-1}\right)$ and $\mathrm{NH}$ bonds $\left(\approx 3336 \mathrm{~cm}^{-1}\right) .{ }^{8}$ The area densities were then obtained by use of IR-scattering cross sections following the work of Lanford and Rand ${ }^{9}$ and of Kim. ${ }^{10}$ Figure 2 shows the development of the absorbance peaks for the sample with the thinnest silicon dioxide layer. The results 


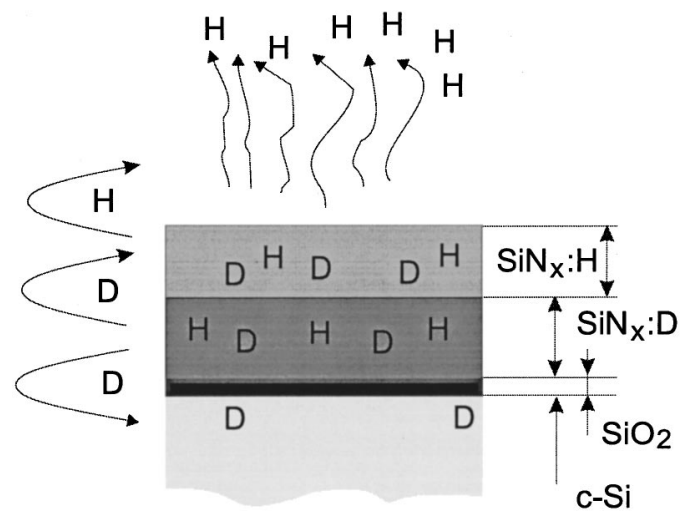

FIG. 1. Stack system for the diffusion experiments.

for the inverse absolute temperature versus bond density reduction for the different oxide thicknesses are displayed in Figs. 3-5. The density reduction $n_{0}-n(T)$ represents the number of ND or NH bonds that have disappeared from the system. The graphs have a logarithmic scale for the density reduction versus the inverse absolute temperature in order to show the correlation of the values with the Boltzmann factor. The SIMS measurements were carried out by Evans East, East Windsor, NJ. ${ }^{11}$ The process involved $3 \mathrm{keV} \mathrm{Cs}{ }^{+}$primary ions that were sputtered at a $60^{\circ}$ angle of incidence. The correlation of the SIMS results with an exact sample depth is difficult since sputtering processes during the measurement process change the location of atoms in the film and the penetration depth of the primary ions has no absolute accuracy. For the observation of the $\mathrm{D}$ diffusion into the $c$-Si it is of crucial importance to know exactly where the inter-

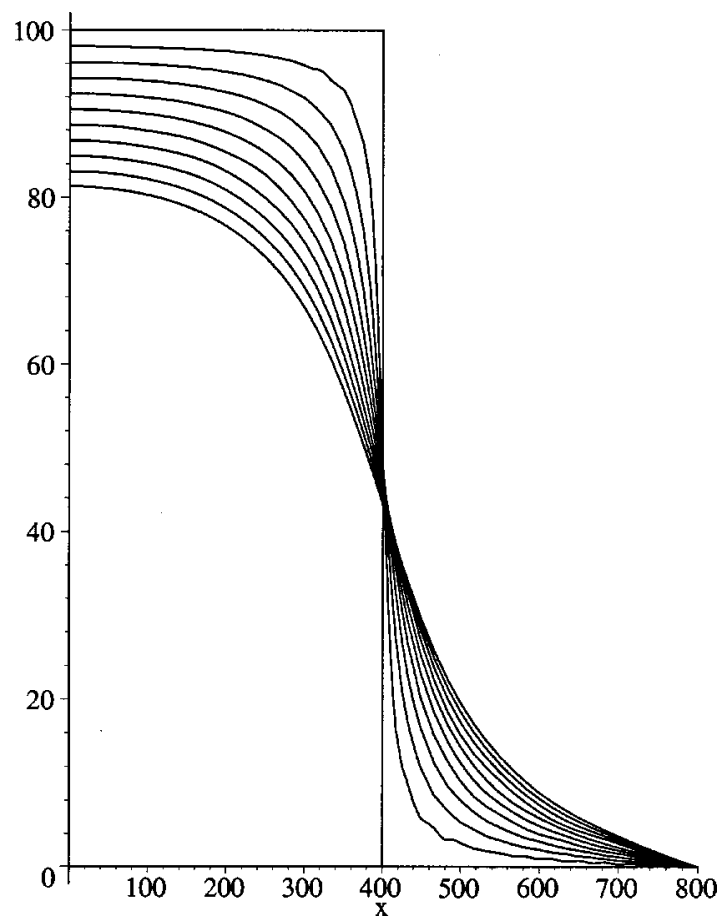

FIG. 2. Theoretical deuterium density profiles of the stack system in $2 \mathrm{~s}$ steps at $900{ }^{\circ} \mathrm{C}$ when atomic diffusion is dominant.

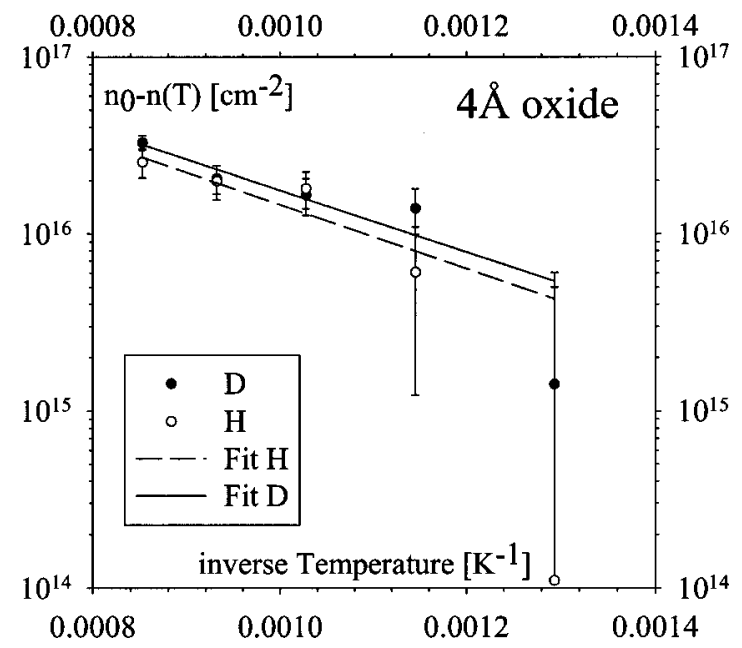

FIG. 3. Inverse temperature dependence of $\mathrm{D}$ and $\mathrm{H}$ area density reduction with a $\mathrm{SiO}_{2}$ layer of $4 \AA$.

face occurs. Therefore, the interface position was located by measuring the $\mathrm{O}$ profile that shows a strong peak near the $c$-Si/silicon nitride interface.

\section{DIFFUSION VERSUS DISSOCIATION MODEL}

Interpretation of the dominant diffusion mechanism from the FTIR data was based on different theoretical predictions of the postanneal profiles of the $\mathrm{Si}_{x} \mathrm{~N}_{y} \mathrm{H}_{z}$ through slow atomic and fast molecular diffusion. While the two different mechanisms do not lead to significantly different net-Hcontent reductions, the depth profiles in both cases can vary profoundly. Diffusion can be described by the diffusion equation,

$$
\left(\frac{\partial}{\partial t}-D \frac{\partial^{2}}{\partial x^{2}}\right) n(x, t)=r(x, t),
$$

which is a result of Fick's law and the continuity equation in one dimension where $n(x, t)$ is the density of the diffusing particles, D the respective diffusion constant in the given silicon nitride system, $x$ and $t$ represent the the film depth

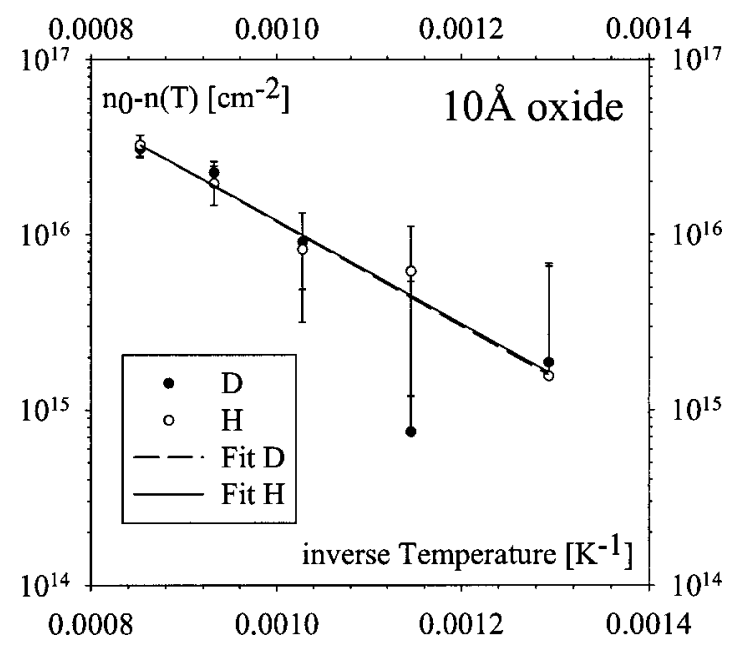

FIG. 4. Inverse temperature dependence of $\mathrm{D}$ and $\mathrm{H}$ area density reduction with a $\mathrm{SiO}_{2}$ layer of $10 \AA$. 


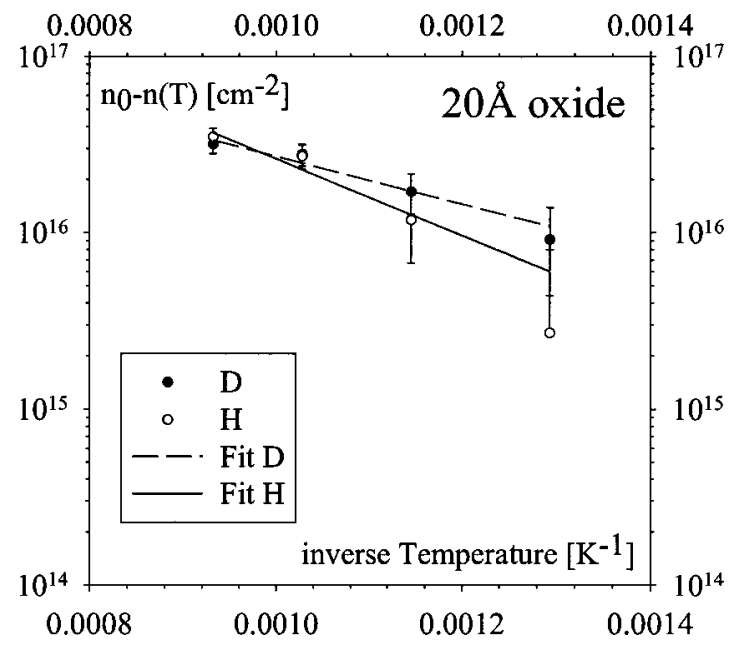

FIG. 5. Inverse temperature dependence of $\mathrm{D}$ and $\mathrm{H}$ area density reduction with a $\mathrm{SiO}_{2}$ layer of $20 \AA$.

and the time, and $r(x, t)$ is a creation and annihilation rate of the diffusing particles. The last vanishes in the case of atomic diffusion. In the case of diffusion of molecular dissociation products, $r(x, t)$ does not vanish, since the molecules can appear or disappear through chemical reactions. This difference between the two mechanisms and the different diffusion constants $\mathrm{D}$ of the $\mathrm{H}$ atoms and the molecules lead to different density depth profiles for the two mechanisms. The slow atomic $\mathrm{H}$ diffusion can sustain a relatively high concentration near the $c$-Si substrate even when most of the $\mathrm{H}$ near the coating surface has already moved into the environment.

The reduction of the $\mathrm{H}$ density during dissociation processes is homogeneous throughout the film since any density gradient is instantly compensated for because of the high diffusivity. This rapid diffusion of dissociated molecules can also cause a fast mixing of different hydrogen isotopes $(\mathrm{H}$ and D) even when these isotopes are spatially separated before the anneal. Chemical dissociation is always accompanied by its reverse reaction, recombination into the network. As long as one region has a lower concentration of a certain isotope than the other layer, the respective dissociation rate is lower than the recombination rate of molecules that have diffused out of the neighboring layer. The density $n(x, t)$ therefore becomes depth independent and the reaction rate $r(x, t)=r(t)$ (=the difference between dissociation and recombination rate) as well. The different depth profiles of atomic diffusion and rapid molecular diffusion mechanisms allow therefore a identification of dominant hydrogen loss mechanisms in the film. In this regard it is important to be aware that slow atomic diffusion can also lead to homogeneous depth profiles if a diffusion barrier at the film surface (e.g., an oxide) prevents atomic recombination into the environment. However, the anneal time needed for the creation of such a homogeneous depth profile due to a surface barrier can be chosen such that the density profile cannot relax into homogeneity even under the worst case assumption, that being that there is no diffusion at all through the surface.

Even though FTIR is not able to measure depth profiles, it was possible to determine the dominant diffusion process with this method by use of two different hydrogen isotopes

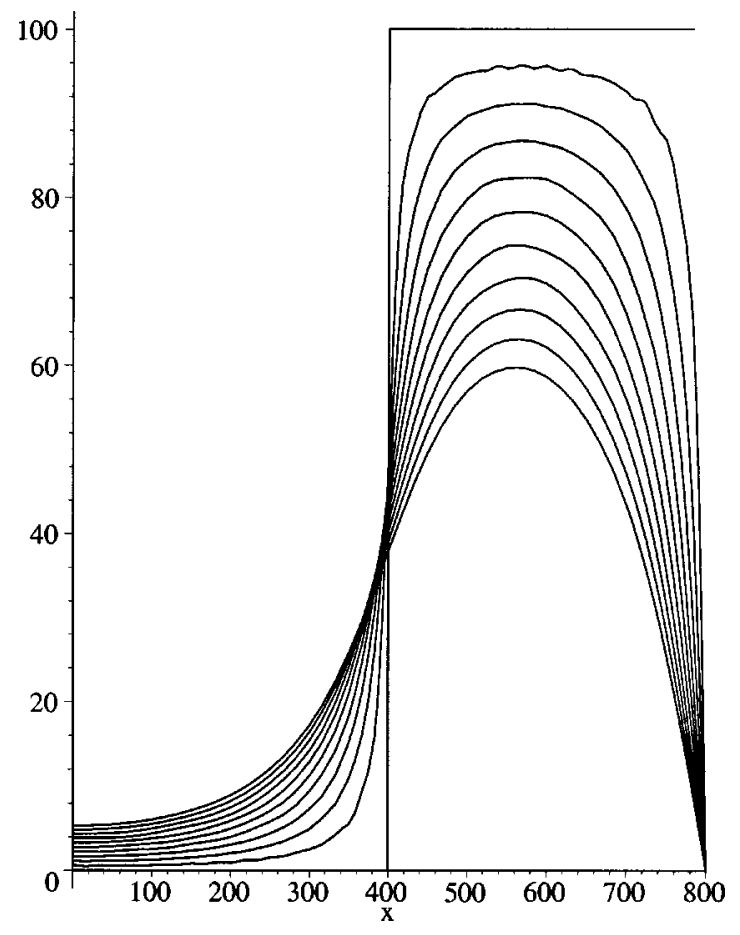

FIG. 6. Theoretical hydrogen density profiles of the stack system in $2 \mathrm{~s}$ steps at $900{ }^{\circ} \mathrm{C}$ when atomic diffusion is dominant.

within the stack. Deuterium in the lower half of the silicon nitride layer works as a labeled substance whose chemical behavior is similar to hydrogen, but is nevertheless distinguishable with spectrometry. If slow atomic diffusion is dominant, the hydrogenated layer acts as a diffusion barrier for the $\mathrm{D}$ atoms by absorbing deuterium from the layer underneath without causing any change in the FTIR spectra. The hydrogen in the top layer however does not have a buffer zone so that a heavy loss of $\mathrm{H}$ out of the system can take place. This leads eventually to a difference between the initially equal area concentrations of D and H. Figures 6 and 7 show this difference between the $\mathrm{D}$ and the $\mathrm{H}$ diffusion in a theoretical "worst case" model whose solutions delivered

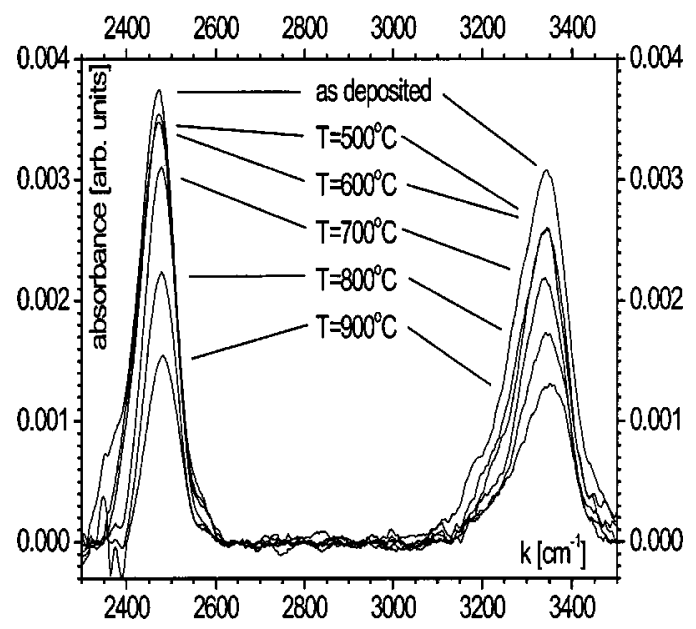

FIG. 7. Postanneal absorbance peaks of ND $\left(\approx 2479 \mathrm{~cm}^{-1}\right)$ and $\mathrm{NH}(\approx 3336$ $\mathrm{cm}^{-1}$ ) bonds. The NH peaks are smaller but also wider, yielding an equal net density of ND and NH bonds. 
an estimate of the highest possible amount of deuterium that could diffuse out of the system with atomic diffusion. The first worst case assumption in this model was atomic diffusion with an activation energy $0.3 \mathrm{eV}$ lower than that calculated by Bik et al. ${ }^{4}$ Second, it is assumed that no $\mathrm{H}$ or D can diffuse into the $c$-Si region. The calculation was carried out by integration of a solution of differential Eq. (1) which had been obtained through expansion of an orthogonal system of functions that was matched to the given boundary conditions. The results were used for the design of the stack system. The profiles clearly show that in case of atomic diffusion a different reduction must occur after an initial equality of $\mathrm{H}$ and $\mathrm{D}$ area density.

\section{DATA ANALYSIS}

The values of the initial concentrations were within their error range at about $6.5 \times 10^{16} \mathrm{~cm}^{-2}$, which confirmed the expected thicknesses of about $400 \AA$ for each silicon nitride layer. Moreover, within the range of the error, both $\mathrm{D}$ and $\mathrm{H}$ layers have initially about the same content. As one can see in the graphs (Figs. 3-5), the concentration losses of both D and $\mathrm{H}$ bonds increase with increasing temperature.

The most interesting result of the diffusion experiment is that the magnitudes of the postanneal densities of $\mathrm{H}$ and $\mathrm{D}$ are in all cases equal within their error ranges. This observation strongly supports the dissociation model. Accordingly, since the slow atomic diffusion process was not significantly determining the reduction of the bond densities, the permeation of $\mathrm{D}$ into the $c$-Si does not seem to be very likely. Furthermore, the results for different oxide thicknesses at equal temperatures are also equal within their error ranges. The oxide layers with different thicknesses were deposited in order to detect possible $\mathrm{H}$ or $\mathrm{D}$ diffusion into the $c$-Si, which could be indicated through higher $\mathrm{D} / \mathrm{H}$ densities in samples with thinner $\mathrm{SiO}_{2}$ barriers. However, this higher density was expected only in the case of the presence of slow D diffusion. For that reason, the reduction independence from oxide thickness also suggests that there is no significant $\mathrm{H}$ permeation from the nitride into the $c$-Si which also strongly supports the notion that the dissociation mechanisms are much more dominant.

In order to see the dependence from the Boltzmann factor, the measured loss function was fitted to the function,

$$
f_{\text {fit }}\left(T^{-1}\right)=a \exp \left(-b T^{-1}\right)
$$

in which the fit parameters are $a$ and $b$. The fit results are displayed in the graphs of Figs. 3-5. Parameter $a$ has only a mathematical but not a physical meaning since it represents the bond loss at infinite temperature. Parameter $b$ however represents the slope of the data points and can therefore be identified with the fraction of the activation energy of the bond reducing processes (whatever that is) and the Boltzmann constant. The results of $b$ for the different fits are displayed in Table I. As one can see, the fit results for the activation energies are between 267 and $590 \mathrm{meV}$. The large scattering of the fit results is due to the large relative error margins of the low temperature $\left(500\right.$ and $\left.600{ }^{\circ} \mathrm{C}\right)$ bond density reduction. In fact, since the exact processes that take
TABLE I. Fit results of density loss function for parameter $b$.

\begin{tabular}{cccc}
\hline \hline $\begin{array}{c}d_{\text {oxide }}(\AA) \\
\AA\end{array}$ & Bond & Fit for $b=E_{a} / k_{B}$ & $\begin{array}{c}E_{a} \\
(\mathrm{eV})\end{array}$ \\
\hline 4 & $\mathrm{ND}$ & $4019 \pm 910$ & $0.346 \pm 0.078$ \\
& $\mathrm{NH}$ & $4167 \pm 1221$ & $0.359 \pm 0.105$ \\
10 & $\mathrm{ND}$ & $6847 \pm 1337$ & $0.590 \pm 0.115$ \\
& $\mathrm{NH}$ & $6778 \pm 599$ & $0.584 \pm 0.052$ \\
20 & $\mathrm{ND}$ & $3099 \pm 582$ & $0.267 \pm 0.050$ \\
& $\mathrm{NH}$ & $5001 \pm 1180$ & $0.430 \pm 0.102$ \\
\hline \hline
\end{tabular}

place in the films are not known, it is questionable whether a constant activation energy throughout the entire temperature range is an appropriate assumption at all. For instance, the 4 and $20 \AA$ samples could also allow a higher slope at lower than at higher temperature which would require a fit with a temperature dependent activation energy.

The interpretation of the observed $\mathrm{H}$ reduction through dissociation into molecules like molecular hydrogen $\left(\mathrm{H}_{2}\right)$ or ammonia $\left(\mathrm{NH}_{3}\right)$ confirms similar observations made by reaction dynamic measurements by Boehme ${ }^{12}$ in simple silicon nitride layers with only hydrogen or deuterium. These measurements also showed the implicitly made assumption that isotopic effects on the diffusion are negligible in the diffusion experiment described above. Moreover, the dissociation hypothesis also confirms spectroscopic data observed by $\mathrm{Zu}$ et al. ${ }^{13}$

The results of the SIMS analysis of the stack confirmed the observations of the FTIR measurements. The profiles of $\mathrm{D}, \mathrm{H}$, and $\mathrm{O}$ (Fig. 8) show the domination of dissociation with fast diffusion reaction products at an anneal at $900{ }^{\circ} \mathrm{C}$. The oxygen density is low in the $c$ - $\mathrm{Si}$ as well as in the center of the silicon nitride at about $400 \AA$ and it is high at the $c$ - $\mathrm{Si}$ interface and also at the surface, which indicates postdeposition ex situ oxidation through exposure to air. The deposited oxide layer at the $c$-Si/nitride interface was thinner than 10 $\AA$. The peak width of the oxide though is at about $150 \AA$. This broadening of the oxide concentration is presumed to be due to oxygen diffusion from the interface layer into the nitride and the silicon regions as well as the finite depth resolution of SIMS, where secondary ions can be scattered not only out of the sample but also into deeper layers causing an error for the measurements of underlying layers.

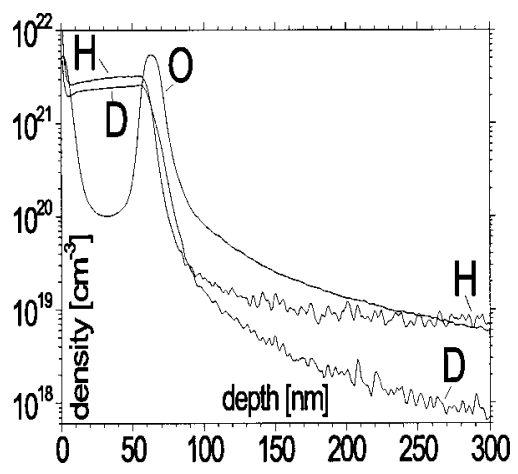

FIG. 8. SIMS profiles of the diffusion stack system after a $900{ }^{\circ} \mathrm{C}$ anneal. The initially different profile shapes for $\mathrm{D}$ and $\mathrm{H}$ are no longer recognizable. 
The most interesting features of the SIMS scan are the D and $\mathrm{H}$ profiles. In spite of a completely different initial distribution, both curves have an almost equal shape and are almost constant throughout the silicon nitride region. The $\mathrm{D}$ curve stretches from about $2.2 \times 10^{21} \mathrm{~cm}^{-3}$ at about $750 \AA$ down to $2 \times 10^{21} \mathrm{~cm}^{-3}$ right below the sample surface. The profile of the hydrogen is proportional to the deuterium profile by a factor of about 1.3 . It starts with $3 \times 10^{21} \mathrm{~cm}^{-3}$ and reduces to about $2.6 \times 10^{21} \mathrm{~cm}^{-3}$ below the surface. The decline of these curves clearly indicates that a slow atomic diffusion process is present in the system. However, this process is of much less importance in comparison with the fast moving dissociation products, which are confirmed by the completely identical shape of the $\mathrm{H}$ and $\mathrm{D}$ profiles and the strong net reduction of $\mathrm{D}$ and $\mathrm{H}$ from the original unannealed sample. While the initial $\mathrm{D}$ and $\mathrm{H}$ concentration was at about $6.5 \times 10^{16} \mathrm{~cm}^{-2}$, the SIMS profiles only total about $1-1.1$ $\times 10^{15} \mathrm{~cm}^{-2}$.

Another feature of the SIMS analysis was the $\mathrm{H}$ and D profile in the $c$-Si below the $c$-Si/oxide/nitride interface. Both $\mathrm{H}$ and $\mathrm{D}$ reduce within $10-20 \mathrm{~nm}$ from about 3 $\times 10^{21} \mathrm{~cm}^{-3}$ by two orders of magnitude down to 3 $\times 10^{19} \mathrm{~cm}^{-3}$. Since the detection limit of $\mathrm{H}$ was 8 $\times 10^{18} \mathrm{~cm}^{-3}$, no statement about the $\mathrm{H}$ profile is possible beyond this limit. However, the $\mathrm{D}$ density remained clearly above its detection limit of $8 \times 10^{17} \mathrm{~cm}^{-3}$ within the first 200 $\mathrm{nm}$ of the $c$-Si bulk. The size and also the shape of this apparent D density indicate that it is caused by SIMS artifacts - D atoms that were sputtered during the measurement from the crater edge with high $\mathrm{D}$ content into the $c-\mathrm{Si}$ crater with low D content. Therefore, significant permeation of $\mathrm{D}$ from the silicon nitride film into the $c$-Si region cannot be confirmed which makes passivation of solar cell emitters through hydrogen from $800 \AA$ antireflective coatings questionable. According to Möller, ${ }^{14}$ the hydrogen density for Si-bulk passivation has to exceed the defect density of the respective bulk material. Even under the assumption that not all of the $\mathrm{D}$ atoms in the $c$-Si region observed by SIMS are artifacts but atoms from the bulk, the densities measured would not exceed the intrinsic bulk defect densities of poly-Si $\left(\leqslant 10^{19}\right)$ substrates and barely even those of monocrystalline Czochralski $\mathrm{Si}\left(\leqslant 10^{18}\right){ }^{14}$ The only region within the $c$-Si where a significant amount of deuterium could be located is within the first $10-20 \mathrm{~nm}$. The profiles show that the $\mathrm{D}$ density in this region is almost an order of magnitude higher than the $\mathrm{H}$ density even though the nitride film contains about the same, and maybe even a little bit more, $\mathrm{H}$ than D. Therefore passivation of the $c$-Si bulk in this region may be possible but the size of it is by far too small for improvement of solar cell emitter regions.

\section{SUMMARY AND CONCLUSIONS}

FTIR and SIMS measurements have shown that the slow atomic diffusion of $\mathrm{H}$ in ammonia rich silicon nitride does exist but the fast diffusion of dissociation products is much more significant. The results strongly suggest that $\mathrm{H}$ permeation into the $c-\mathrm{Si}$ region through annealing is unlikely, therefore improvement of $c$-Si solar cells by hydrogen bulk passivation is very unlikely during the postdeposition anneal of AR coatings.

In order to make a better statement about the passivation of $c$-Si cells, electronical measurements are necessary as well as a deeper understanding of the dissociation processes that explain which dissociation reactions take place at different rates and temperatures.

${ }^{1}$ F. Duerinckx, J. Szlufcik, A. Ziebakowski, J. Nijs, and R. Mertens, Proceedings of 14th European Photovoltaic Solar Energy Conference, Barcelona, 1997.

${ }^{2}$ F. Duerinckx et al., Proceedings of 13th European Photovoltaic Solar Energy Conference, Nice, 1996.

${ }^{3}$ J. Szlufcik et al., Proceedings of 12th European Photovoltaic Solar Energy Conference, Amsterdam, 1994.

${ }^{4}$ W. M. Arnold Bik, R. N. H. Linnsen, F. H. P. M. Habraken, and W. F. van der Weg, Appl. Phys. Lett. 56, 2530 (1990).

${ }^{5}$ H. J. Stein and H. A. R. Wegener, J. Electrochem. Soc. 124, 908 (1977).

${ }^{6}$ G. Lucovsky, P. D. Richard, D. V. Tsu, S. Y. Lin, and R. J. Markunas, J. Vac. Sci. Technol. A 4, 681 (1986).

${ }^{7}$ G. Bruno, P. Capezutto, and A. Madan, Plasma Deposition of Amorphous Silicon Based Materials (Academic, Boston, 1995).

${ }^{8} \mathrm{~S}$. Pinchas and I. Laulicht, Infrared Spectra of Labelled Compounds (Academic, London, 1971), p. 8.

${ }^{9}$ W. A. Lanford and M. J. Rand, J. Appl. Phys. 49, 2473 (1978).

${ }^{10}$ S. S. Kim, Ph.D. thesis, North Carolina State University, 1990.

${ }^{11}$ M. S. Denker, Secondary Ion Mass Spectroscopy Analytical Report, Technical Report No. EE 99-2250, Evans East, 104 Windsor Center, Suite 101, East Windsor, NJ 08520, 1999.

${ }^{12}$ C. Boehme, Diploma thesis, Ruprecht-Karls-Universität, Heidelberg, 2000.

${ }^{13}$ Z. Zu, G. Santos-Filho, G. Stevens, M. J. Williams, and G. Lucovsky, J. Vac. Sci. Technol. A 13, 607 (1995).

${ }^{14}$ H. J. Möller, Semiconductors for Solar Cells (Artech House, Boston, 1993). 